\title{
The efficacy and safety of co-administration of fimasartan and rosuvastatin to patients with hypertension and dyslipidemia
}

Moo-Yong Rhee ${ }^{1}$, Taehoon Ahn², Kiyuk Chang ${ }^{3}$, Shung Chull Chae ${ }^{4}$, Tae-Hyun Yang ${ }^{5}$, Wan Joo Shim Tae Soo Kang ${ }^{7}$, Jae-Kean Ryu ${ }^{8}$, Deuk-Young Nah', Tae-Ho Park ${ }^{10}$, In-Ho Chae ${ }^{11}$, Seung Woo Park ${ }^{12}$, Hae-Young Lee ${ }^{13}$, Seung-Jea Tahk ${ }^{14}$, Young Won Yoon ${ }^{15}$, Chi Young Shim ${ }^{16}$, Dong-Gu Shin ${ }^{17}$, Hong Seog Seo ${ }^{18}$, Sung Yun Lee ${ }^{19}$, Doo II Kim² ${ }^{20}$, Jun Kwan ${ }^{21}$, Seung-Jae Joo ${ }^{22}$, Myung Ho Jeong ${ }^{23}$, Jin-Ok Jeong ${ }^{24}$, Ki Chul Sung ${ }^{25}$, Seok Yeon Kim ${ }^{26}$, Sang-Hyun Kim ${ }^{27}$, Kook-Jin Chun ${ }^{28}$ and Dong Joo Oh ${ }^{18^{*}}$

\begin{abstract}
Background: Hypertension and dyslipidemia are major risk factors of cardiovascular disease (CVD) events. The objective of this study was to evaluate the efficacy and safety of the co-administration of fimasartan and rosuvastatin in patients with hypertension and hypercholesterolemia.
\end{abstract}

Methods: We conducted a randomized double-blind and parallel-group trial. Patients who met eligible criteria after 4 weeks of therapeutic life change were randomly assigned to the following groups.

1) co-administration of fimasartan $120 \mathrm{mg} /$ rosuvastatin $20 \mathrm{mg}$ (FMS/RSV), 2) fimasartan $120 \mathrm{mg}$ (FMS) alone 3) rosuvastatin $20 \mathrm{mg}$ (RSV) alone. Drugs were administered once daily for 8 weeks.

Results: Of 140 randomized patients, 135 for whom efficacy data were available were analyzed. After 8 weeks of treatment, the FMS/RSV treatment group showed greater reductions in sitting systolic (siSBP) and diastolic (siDBP) blood pressures than those in the group receiving RSV alone (both $p<0.001$ ). Reductions in siSBP and siDBP were not significantly different between the FMS/RSV and FMS alone groups ( $p=0.500$ and $p=0.734$, respectively). After 8 weeks of treatment, FMS/RSV treatment showed greater efficacy in percentage reduction of low-density lipoprotein cholesterol (LDL-C) level from baseline than that shown by FMS alone treatment $(p<0.001)$. The response rates of siSBP with FMS/RSV, FMS alone, and RSV alone treatments were 65.22, 55.56, and 34.09\%, respectively (FMS/RSV vs. RSV, $p=0.006$ ). The LDL-C goal attainment rates with FMS/RSV, RSV alone, and FMS alone treatments were $80.43 \%, 81.82 \%$, and $15.56 \%$, respectively (FMS/RSV vs. FMS, $p<0.001$ ). Incidence of adverse drug reactions with FMS/RSV treatment was $8.33 \%$, which was similar to those associated with FMS and RSV alone treatments.

Conclusion: This study demonstrated that the co-administration of fimasartan and rosuvastatin to patients with both hypertension and hypercholesterolemia was efficacious and safe.

Trial registration: ClinicalTrials.gov Identifier: NCT02166814. 16 June 2014

Keywords: Fimasartan, Rosuvastatin, Hypertension, Hypercholesterolemia

\footnotetext{
* Correspondence: ohdj@korea.ac.kr

${ }^{18}$ Division of Cardiology, Korea University Guro Hospital, 148, Gurodong-ro,

Guro-gu, Seoul 08308, Republic of Korea

Full list of author information is available at the end of the article
} 


\section{Background}

Hypertension and hypercholesterolemia are major risk factors of cardiovascular disease (CVD) events. The coexistence of both risk factors is quite common. The prevalence of coexistence was estimated to be $30 \%$ in an epidemiologic study [1]. The co-existence of hypertension and hypercholesterolemia can act additively or synergistically to elevate CVD risk [2, 3]. Because of the increased risk of CVD with comorbidities, guidelines have recommended simultaneous treatment of both risk factors $[4,5]$. Indeed, long-term reduction of both serum total cholesterol (TC) and systolic blood pressure (SBP) by $10 \%$ could reduce major CVD events by $45 \%$ [6].

The beneficial effects for the prevention of CVD events in most clinical trials have been obtained from controlled adherence to study drugs. Poor adherence to treatment is a problem in real practice, leading to increased cardiovascular disease events $[7,8]$. To improve adherence to drug treatment, regimen simplification by reducing the number of drugs and the frequency of dosing has been found to be effective. Single pill combination is one of the methods that can simplify regimens and enhance adherence to treatment $[9,10]$.

The present study was a phase III trial to evaluate the efficacy and safety of the co-administration of fimasartan and rosuvastatin in patients with both hypertension and hypercholesterolemia.

\section{Methods}

\section{Patients}

Patients (age 20-75 years) with hypertension (blood pressure $\geq 140 / 90 \mathrm{~mm} \mathrm{Hg}$ or currently on antihypertensive medication) and dyslipidemia (defined in accordance with the National Cholesterol Education Program Adult Panel III (NCEP-ATP III) [11] or currently on lipid modifying medications) were included. Exclusion criteria were a mean sitting SBP $(\mathrm{siSBP}) \geq 180 \mathrm{mmHg}$ at screening visit and/or sitting diastolic blood pressure (siDBP) $\geq$ $110 \mathrm{mmHg}$; differences between arms $\geq 20 \mathrm{mmHg}$ for siSBP or $\geq 10 \mathrm{mmHg}$ for siDBP; secondary hypertension; secondary dyslipidemia (nephrotic syndrome, dysproteinemia, Cushing's syndrome, and obstructive hepatopathy); fasting triglyceride level at pre-randomization visit $\geq$ $400 \mathrm{mg} / \mathrm{dL}$; history of myopathy, rhabdomyolysis, and/or creatinine kinase $\geq 2 \times$ upper limit of normal; history of hypersensitivity to angiotensin receptor antagonist and/or 3-hydroxy-3-methylglutaryl coenzyme A (HMG-CoA) reductase inhibitors; gastrointestinal surgery or active inflammatory gastrointestinal diseases potentially affecting study drug absorption in the preceding 12 months; uncontrolled (glycated hemoglobin $>9 \%$ at pre-randomization visit) or insulin-dependent diabetes mellitus; liver disease (aspartate aminotransferase and/or alanine aminotransferase $\geq 2 \times$ upper normal limit); hepatitis B (including positive test for $\mathrm{HBsAg}$ ) or hepatitis C-positive; impaired function of kidney (serum creatinine $\geq 1.5 \times$ upper normal limit); human immunodeficiency virus infection; electrolyte imbalance (sodium level $<133 \mathrm{mmol} / \mathrm{L}$ or $\geq$ $145 \mathrm{mmol} / \mathrm{L}$ or potassium level $<3.5 \mathrm{mmol} / \mathrm{L}$ or $\geq$ $5.5 \mathrm{mmol} / \mathrm{L})$; retinal hemorrhage; visual disturbance or retinal microaneurysm within the past 6 months; history of abusing drugs or alcohol; ischemic heart disease within the previous 6 months (angina pectoris, acute myocardial infarction); peripheral vascular disease); percutaneous coronary intervention, or coronary artery bypass graft within the previous 6 months; severe cerebrovascular disease within previous 6 months (cerebral infarction, or cerebral hemorrhage); New York Heart Association functional class III and VI heart failure; clinically significant cardiac arrhythmia; or history of any type of malignancy within the previous 5 years; women in pregnancy, breastfeeding, or child-bearing potential without no intention of using a contraceptive.

\section{Study design}

This multicenter, randomized, double-blind, and parallelgroup trial was performed at 29 study centers in Korea. Institutional Review Board of the participating institution (Additional file 1: Table S1) and the Ministry of Food and Drug Safety approved the study design. Written informed consent was obtained from all patients. After screening, patient who met the eligible criteria entered 4 weeks of therapeutic life changes (TLC) consisting of detailed education given by a study coordinator. During the 4 weeks of TLC, patients who were already receiving lipid modifying and/or antihypertensive medications discontinued taking their lipid modifying medications for at least 4 weeks and antihypertensive medications for at least 2 weeks prior to randomization. After 4 weeks of TLC, patients who met the inclusion criteria for randomization (Table 1) were randomly assigned at a 1:1:1 ratio to receive one of three treatments once daily for 8 weeks: 1) fimasartan $120 \mathrm{mg} /$ rosuvastatin $20 \mathrm{mg}$ (FMS/RSV); 2) fimasartan $120 \mathrm{mg}$ (FMS); 3) rosuvastatin $20 \mathrm{mg}$ (RSV) using a sealed envelope with the randomization number. Study drugs were supplied by Boryung Pharmaceutical Co. Ltd. (Seoul, Republic of Korea). Randomization criteria of dyslipidemia were based on NCEP-ATP III [11].

All patients were instructed to orally take the assigned drug once daily in the morning for the study duration. Prior to a scheduled visit, patients were instructed to fast $12 \mathrm{~h}$ without taking the study drug in the morning. At each visit, three measurements of siSBP, siDBP, and pulse rate were taken from the reference arm after a 5 min rest and with a 2 min interval between measurements using a semi-automated sphygmomanometer [HEM-7080IT, Omron Corporation, Kyoto, Japan] [12, 13]. The three siSBP and siDBP measurements were averaged. 
Table 1 Inclusion criteria at randomization

\begin{tabular}{llll}
\hline Risk category & Cardiovascular risk factors $^{\mathrm{a}}$ & Randomization criteria \\
\cline { 3 - 4 } & & LDL-cholesterol $(\mathrm{mg} / \mathrm{dL})$ & Averaged sitting SBP $(\mathrm{mmHg})$ \\
\hline Low risk & 0 risk factors & $\geq 160$ and $\leq 250$ & $\geq 140$ and $<180$ \\
Moderate risk & $1+$ risk factors and 10 year risk $<10 \%$ & $\geq 160$ and $\leq 250$ & \\
Moderate high risk & $1+$ risk factors and 10-year risk from 10\% to 20\% & $\geq 100$ and $\leq 250$ \\
High risk & $\mathrm{CHD}^{\mathrm{b}}$ and CHD risk equivalent ${ }^{\mathrm{c}}$ & &
\end{tabular}

${ }^{a}$ Risk factors: include cigarette smoking, hypertension ( $\mathrm{BP} \geq 140 / 90 \mathrm{~mm} \mathrm{Hg}$ or on antihypertensive medication), low HDL cholesterol ( $\left.<40 \mathrm{mg} / \mathrm{dL}\right)$, family history of premature CHD (CHD in male first-degree relative $<55$ years of age; CHD in female first-degree relative $<65$ years of age), and age (men $\geq 45$ years; women $\geq 55$ years) ${ }^{b} \mathrm{CHD}$ (coronary heart disease) includes history of myocardial infarction, unstable angina, stable angina, coronary artery procedures (angioplasty or bypass surgery), or evidence of clinically significant myocardial ischemia

${ }^{\mathrm{C}} \mathrm{CHD}$ (coronary heart disease) risk equivalents include clinical manifestations of noncoronary forms of atherosclerotic disease (peripheral arterial disease, abdominal aortic aneurysm, and carotid artery disease [transient ischemic attacks or stroke of carotid origin or $>50 \%$ obstruction of a carotid artery]), diabetes, and $2+$ risk factors with 10-year risk for hard CHD > 20\%

Fasting blood samples obtained during scheduled visits were sent to the central laboratory (Seoul Medical Science Institute, Seoul, Korea) for analysis of TC, triglyceride, high-density lipoprotein cholesterol (HDL-C), and low density lipoprotein cholesterol (LDL-C) levels.

\section{Efficacy evaluation}

The primary efficacy points were comparing: 1) changes from baseline in mean siSBP after 8 weeks of treatment between the FMS/RSV and RSV treatment groups, and 2) percentage change from baseline of mean LDL-cholesterol after 8 weeks of treatment between the FMS/RSV and FMS treatment groups.

The secondary efficacy points were comparing: 1) changes from baseline in mean siSBP after 8 weeks of treatment between the FMS/RSV and FMS alone treatment groups, 2) percentage change from baseline of LDLcholesterol after 8 weeks of treatment between the FMS/ RSV and RSV treatment groups, 3) changes from baseline in TC, HDL-C, and triglyceride levels after 8 weeks of treatment, 4) changes from baseline in mean siDBP after 8 weeks of treatment, 5) blood pressure control rate (the percentage of patients who reached mean siSBP < $140 \mathrm{mmHg}$ after 8 weeks of treatment) and response rate (the percentage of patients who reached a mean siSBP $<$ $140 \mathrm{mmHg}$ and/or a reduction of siSBP $\geq 20 \mathrm{mmHg}$ from baseline values after 8 weeks of treatment), and 6) the percentage of patients achieving the LDL-C target level after 8 weeks of treatment (goal attainment rate) according to the NCEP-ATP III guidelines (high risk: LDL-C level $<100 \mathrm{mg} / \mathrm{dL}$; moderate/moderate high risk: LDL-C level < 130 mg/dL; low risk: LDL-C level < 160 mg/dL) [11].

\section{Safety evaluation}

Assessment of safety and tolerability were conducted by medical examination, patient reporting, and laboratory tests (electrocardiography at baseline and the end of week 8 , blood and urine tests at baseline and the end of week 8 , pregnancy test at every visit). All adverse events (occurrence and elimination dates, detailed nature, duration, seriousness, intensity, significance, and relationship to the study drug) occurring during the study period were recorded.

\section{Sample size}

This was a therapeutic confirmatory study to verify the superiority of FMS/SRV treatment in terms of change in mean siSBP (from baseline to week 8) compared to RSV alone, and in terms of percentage change of LDL-C (from baseline to week 8) compared to FMS alone. Therefore, two statistical hypotheses were formulated and the number of subjects was calculated. Total test power for the whole hypothesis was set to $80 \%$ while the two-sided significance level of each hypothesis was set to $5 \%$. The test power for each hypothesis was set to $90 \%$ without adjusting multiplicity.

It was assumed that the mean change in siSBP with the FMS/RSV treatment was identical to that of the FMS alone treatment of a previous study, and that the mean change in siSBP of the RSV alone treatment was identical to that of the placebo. The difference in the mean and the standard deviation between the two treatment groups were estimated using weighted mean and pooled standard deviation based on the results of previous studies [14, 15]. The mean siSBP was lowered $-17.41 \mathrm{mmHg}$ by FMS treatment and $-7.34 \mathrm{mmHg}$ by placebo. The difference in siSBP lowering effect between the two groups was $10.07 \mathrm{mmHg}$ with a pooled standard deviation of $12.91 \mathrm{mmHg}$. Required sample sizes were at least 36 subjects per group. A total of 135 subjects (45 subjects each for 3 groups) were considered in order to make the sample size cutoff, working under the assumption of a dropout rate of $20 \%$.

The mean percent change of LDL-C in previous studies was $-57.0 \%$ in the RSV treatment alone and $-3.6 \%$ in the placebo. The difference in LDL-C lowering effect between the two groups was $53.4 \%$ with the standard deviation assumed to be $20 \%$ [16]. Required sample sizes in the comparison of the LDL-C lowering effect were at least 4 
subjects per group. Therefore, a total of 15 subjects (3 groups) were required under the assumption of a dropout rate of $20 \%$.

Finally, a total of 135 subjects were chosen as the sample size because the sample size required for the comparison of the siSBP lowering effect was greater than that required for the comparison of the LDL-C lowering effect.

\section{Statistical analyses}

For the main analysis of efficacy, full analysis set (FAS) was used while per-protocol set (PPS) analysis was additional. FAS included all subjects with at least one efficacy evaluation result after baseline. Within the FAS, PPS consisted of patients who completed the treatment course without any significant protocol violations that might affect efficacy outcomes. If any values in the primary and secondary efficacy points were missed, the Last-Observation-Carried-Forward imputation method was used. The response rate and control rate were analyzed by using the Non-Response Imputation method to process patients with missing data at the time point of measurement as a non-responder.

The safety set included the patients who had been administered the investigational product at least once after randomization and had been assessed for safety at least once. The safety analysis was conducted based on the actual treatment group, regardless of the randomized group. Safety assessment variables for missed data were not imputed.

Using baseline values (blood pressure and LDL-C), age, gender, and smoking status as covariates, analysis of covariance (ANCOVA) was performed to test the difference in the two primary efficacy endpoints between groups at $5 \%$ significance level (two-sided). In order to test for differences within the treatment group, one sample $t$ test for the percent change in LDL-C and paired $t$-test for the change in siSBP were performed. If normality was not satisfied, a Wilcoxon signed rank test was performed.

Descriptive statistics for the secondary efficacy endpoints were presented for treatment groups. One sample $t$-test for the percentage change of LDL-C, TC, HDL-C and TG from baseline within the treatment group, and paired $t$-test for the change in siSBP and siDBP were performed. If normality was not satisfied, Wilcoxon signed rank test was performed.

The proportion and 95\% confidence interval for the response rate and control rate of siSBP, as well as the goal attainment rate for LDL-C at week 8 were presented. Using the treatment groups as factors and baseline data, age, gender and smoking status as covariates, the logistic regression analysis was performed for the significant difference between monotherapy and combination therapy group.

Additionally, the interactive effect was tested by using an ANCOVA model in order to confirm the existence of an interaction effect between the monotherapy and combination treatment groups by variables. The combination therapy group was determined to be superior to the monotherapy group if both variables in the combination therapy group showed statistically significant superiority to the monotherapy group as a result of an ANCOVA test.

Medical Dictionary for Regulatory Activities (MedDRA; $\mathrm{v}$ 18.0) was used in coding adverse events. The percentage of patients who experienced any adverse events between groups was compared using Chi-square or Fisher's exact tests. Incidence of adverse events was presented according to severity and relationship with study drugs.

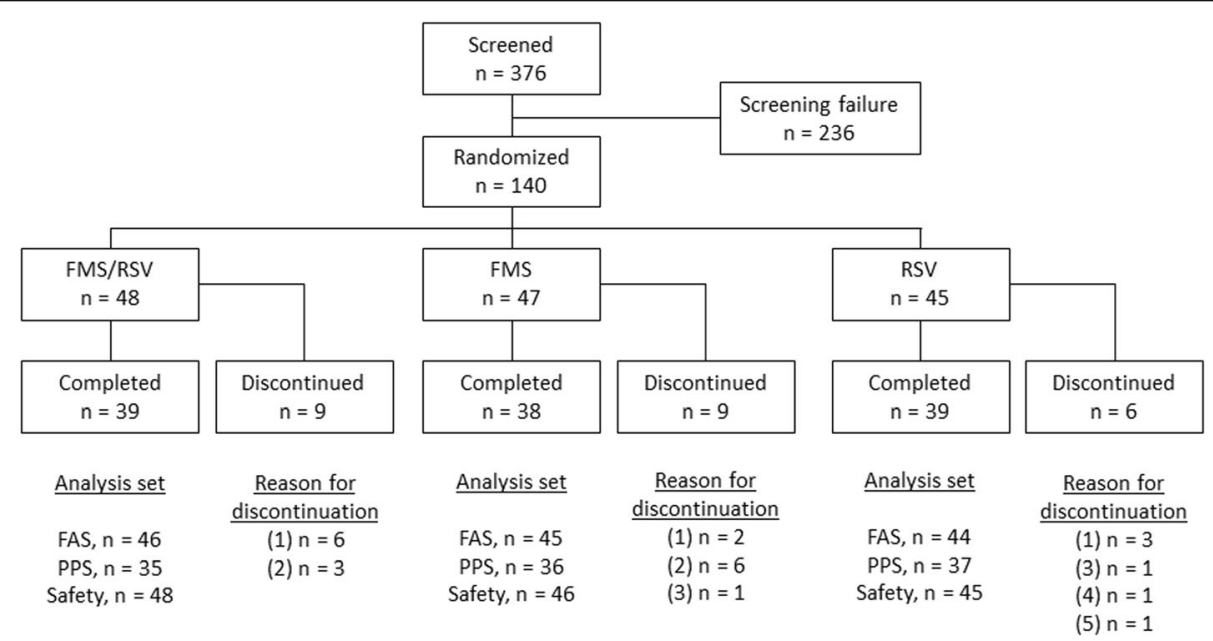

Fig. 1 Subject disposition and reasons for study discontinuation. Reasons for discontinuation included (1) withdrawal of consent, (2) protocol violations, (3) lack of efficacy, (4) adverse events, and (5) other reasons. FMS: fimasartan; RSV, rosuvastatin. FMS/RSV: fimasartan 120 mg/rosuvastatin $20 \mathrm{mg}$ treatment; FMS: fimasartan $120 \mathrm{mg}$ alone treatment; RSV: rosuvastatin $20 \mathrm{mg}$ alone treatment; FAS: full analysis set; PPS: per-protocol set 
Baseline characteristics were compared among the treatment groups using Kruskal-Wallis test for continuous variables and chi-square test for categorical variables. All statistical analyses were performed using $\mathrm{SAS}^{\circ}$ software (v 9.3; SAS Institute, Cary, NC, USA).

\section{Results}

\section{Patients' disposition}

Among 376 screened patients, 140 were assigned to 8-week treatments of FMS/RSV, FMS alone, or RSV alone. After randomization, 24 patients discontinued the study owing to consent withdrawal $(n=11)$, protocol violation $(n=9)$, lack of efficacy $(n=2)$, adverse events $(n=1)$, and other reasons $(n=1)$ (Fig. 1). Of the 140 randomized patients, 135 were included in primary efficacy analysis after excluding five patients owing to missing efficacy data (Table 2). Their mean age was $60.5 \pm 8.7$ years. The majority of the study population comprised men (73.3\%). The body mass index of the FMS/RSV treatment group and siDBP of the RSV alone treatment group were the highest among the three groups. Other baseline characteristics were not significantly different among the groups. Lipid-modifying agents were taken by 93 patients (68.9\%). Angiotensinconverting enzyme inhibitors or angiotensin receptor blockers were taken by 81 patients (60.0\%).

\section{Efficacy}

Changes in siSBP, siDBP and LDL-C are presented in Table 3. FMS/RSV combination treatment had greater

Table 2 Baseline characteristics of the study population

\begin{tabular}{|c|c|c|c|c|c|}
\hline Demographics & $\begin{array}{l}\text { Total } \\
(n=135)\end{array}$ & $\begin{array}{l}\text { FMS/RSV } \\
(n=46) \\
\end{array}$ & $\begin{array}{l}\text { FMS } \\
(n=45)\end{array}$ & $\begin{array}{l}\text { RSV } \\
(n=44)\end{array}$ & $P$-value \\
\hline Age (year) & $60.5(8.7)$ & $59.3(8.7)$ & $62.3(9.5)$ & $59.9(7.7)$ & $0.137^{\mathrm{a}}$ \\
\hline Sex, men, n (\%) & $99(73.3)$ & $31(67.4)$ & $34(75.6)$ & $34(77.3)$ & $0.524^{b}$ \\
\hline Body mass index $\left(\mathrm{kg} / \mathrm{m}^{2}\right)$ & $25.6(2.7)$ & $26.4(2.8)$ & $25.1(2.6)$ & $25.4(2.8)$ & $0.033^{\mathrm{a}}$ \\
\hline \multicolumn{6}{|l|}{ Baseline blood pressure, $\mathrm{mmHg}$} \\
\hline Systolic & $152.8(9.5)$ & $152.5(9.9)$ & $151.3(9.0)$ & $154.7(9.5)$ & $0.157^{\mathrm{a}}$ \\
\hline Diastolic & $89.4(9.1)$ & $89.4(8.3)$ & $85.8(9.3)$ & $93.1(8.5)$ & $0.001^{\mathrm{a}}$ \\
\hline Baseline Pulse Rate (beats/min) & $75.2(12.1)$ & $76.4(13.1)$ & $73.0(10.9)$ & $76.2(12.3)$ & $0.441^{a}$ \\
\hline Baseline LDL-C (mg/dL) & $165.7(34.6)$ & $171.3(36.0)$ & $164.1(39.6)$ & $161.4(26.7)$ & $0.294^{\mathrm{a}}$ \\
\hline \multicolumn{6}{|l|}{ Smoking, n (\%) } \\
\hline Current Smoker & $37(27.4)$ & $14(30.4)$ & $12(26.7)$ & $11(25.0)$ & \multirow[t]{3}{*}{$0.933^{b}$} \\
\hline Non-smoker & $57(42.2)$ & $17(37.0)$ & $20(44.4)$ & $20(45.5)$ & \\
\hline Ex-smoker & $41(30.4)$ & $15(32.6)$ & $13(28.9)$ & 13 (29.6) & \\
\hline \multicolumn{6}{|l|}{ Drinking, n (\%) } \\
\hline Current drinker & $83(61.5)$ & $30(65.2)$ & $27(60.0)$ & $26(59.1)$ & \multirow[t]{2}{*}{$0.811^{b}$} \\
\hline Non-drinker & $52(38.5)$ & $16(34.8)$ & $18(40.0)$ & $18(40.9)$ & \\
\hline \multicolumn{6}{|c|}{ Medication history of cardiovascular system, n (\%) } \\
\hline Lipid modifying agents & $93(68.9)$ & $31(67.4)$ & $34(75.6)$ & $28(63.6)$ & \\
\hline ACE inhibitors or ARBs & $81(60.0)$ & $28(60.9)$ & $29(64.4)$ & $24(54.6)$ & \\
\hline Calcium channel blockers & $38(28.2)$ & $9(19.6)$ & $13(28.9)$ & $16(36.4)$ & \\
\hline Beta blockers & $10(7.4)$ & $1(2.2)$ & $4(8.9)$ & $5(11.4)$ & \\
\hline Cardiac drugs & $7(5.2)$ & - & $5(11.1)$ & $2(4.6)$ & \\
\hline Diuretics & $5(3.7)$ & $1(2.2)$ & $2(4.4)$ & $2(4.6)$ & \\
\hline Peripheral vasodilators & $4(3.0)$ & $1(2.2)$ & $3(6.7)$ & - & \\
\hline Vasoprotectives & $1(0.7)$ & - & - & $1(2.3)$ & \\
\hline \multicolumn{6}{|l|}{ Medical history, n (\%) } \\
\hline Diabetes mellitus & $33(24.4)$ & $8(17.4)$ & 16 (35.6) & $9(20.5)$ & \\
\hline Angina pectoris & $6(4.4)$ & $1(2.2)$ & $2(4.4)$ & $3(6.8)$ & \\
\hline
\end{tabular}

Data are expressed as mean and standard deviation in parenthesis, and number and percent in parenthesis FMS/RSV fimasartan $120 \mathrm{mg} /$ rosuvastatin $20 \mathrm{mg}$ treatment, FMS fimasartan $120 \mathrm{mg}$ alone treatment, RSV rosuvastatin $20 \mathrm{mg}$ alone treatment, $L D L-C$ low-density lipoprotein cholesterol

Difference among treatment groups: ${ }^{a}$ Kruskal-Wallis test for continuous variables, ${ }^{\text {b }}$ chi-square test for categorical variables 
Table 3 Changes in sitting systolic blood pressure, sitting diastolic blood pressure and low-density lipoprotein cholesterol at Week 8 from baseline

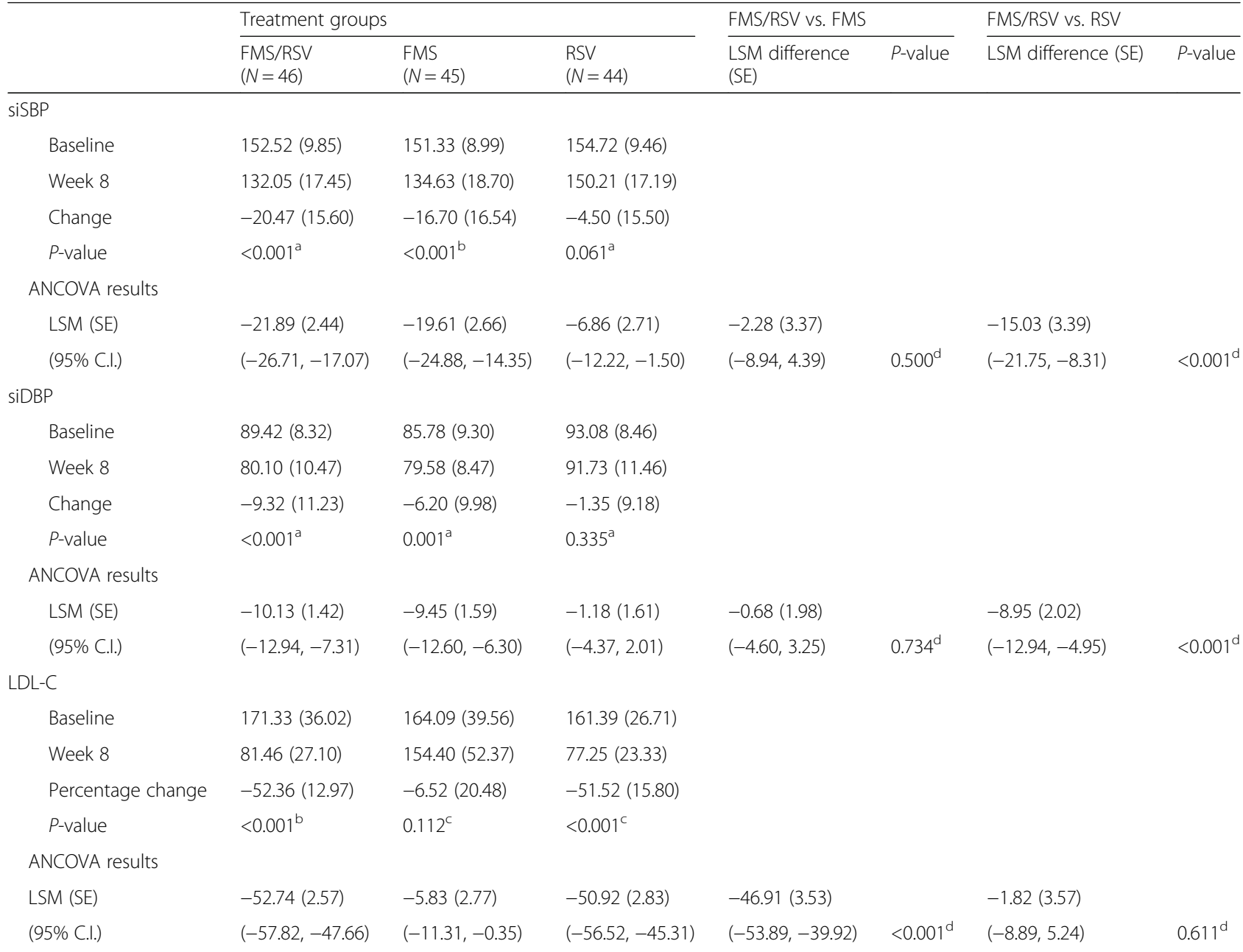

FMS/RSV fimasartan $120 \mathrm{mg} /$ rosuvastatin $20 \mathrm{mg}$ treatment, FMS fimasartan $120 \mathrm{mg}$ alone treatment, $R S V$ rosuvastatin $20 \mathrm{mg}$ alone treatment, $L D L-C$ low-density lipoprotein, siSBP sitting systolic blood pressure, siDBP sitting diastolic blood pressure, SD standard deviation, $L S M$ least square mean, SE standard error, ANCOVA analysis of covariance

Percent change from baseline was compared by ${ }^{a}$ paired $t$-test, ${ }^{b}$ one sample $t$-test, or ${ }^{c}$ Wilcoxon signed rank test

${ }^{\mathrm{d}}$ Comparison between the combination therapy and monotherapy was analyzed by ANCOVA model adjusted for baseline values, age, gender and smoking status

efficacy in reducing siSBP from baseline after 8 weeks of treatment compared to that reported for RSV alone treatment $(p<0.001)$. Changes of siSBP was not significantly different between FMS/RSV and FMS alone groups $(p=0.500)$. Likewise, the reduction in siDBP from baseline after 8 weeks of treatment was significantly larger in the FMS/RSV treatment group compared to that in the RSV alone treatment group $(p<0.001)$. FMS/RSV and FMS alone treatments were not significantly different in the reduction of $\operatorname{siDBP}(p=0.734)$. The least square mean (LSM) difference in siSBP and siDBP between FMS/RSV and RSV alone treatment groups was $-15.03 \mathrm{mmHg}$ (95\% confidence interval: -21.75 to $-8.31 \mathrm{mmHg}$ ) and $-8.95 \mathrm{mmHg}(95 \%$ confidence interval: -12.94 to $-4.95 \mathrm{mmHg}$ ), respectively.
The percentage change of LDL-C from baseline to after 8 weeks of treatment was larger in the FMS/RSV treatment group than in the FMS alone treatment group $(p<0.001)$. The percentage change of LDL-C between the FMS/RSV and RSV alone treatment groups was not different $(p=0.611)$. The LSM difference of LDL-C percentage change between FMS/RSV and FMS alone treatment groups was $-46.91 \%$ (95\% confidence interval: -53.89 to $-39.92 \%)$.

The response rate of siSBP, control rate of siSBP, and goal attainment rate of LDL-C in FAS after 8 weeks of treatment are presented in Fig. 2. The response rate of siSBP in the FMS/RSV treatment, FMS alone treatment, and RSV alone treatment groups was 65.22, 55.56, and $34.09 \%$, respectively (FMS/RSV vs. RSV, 


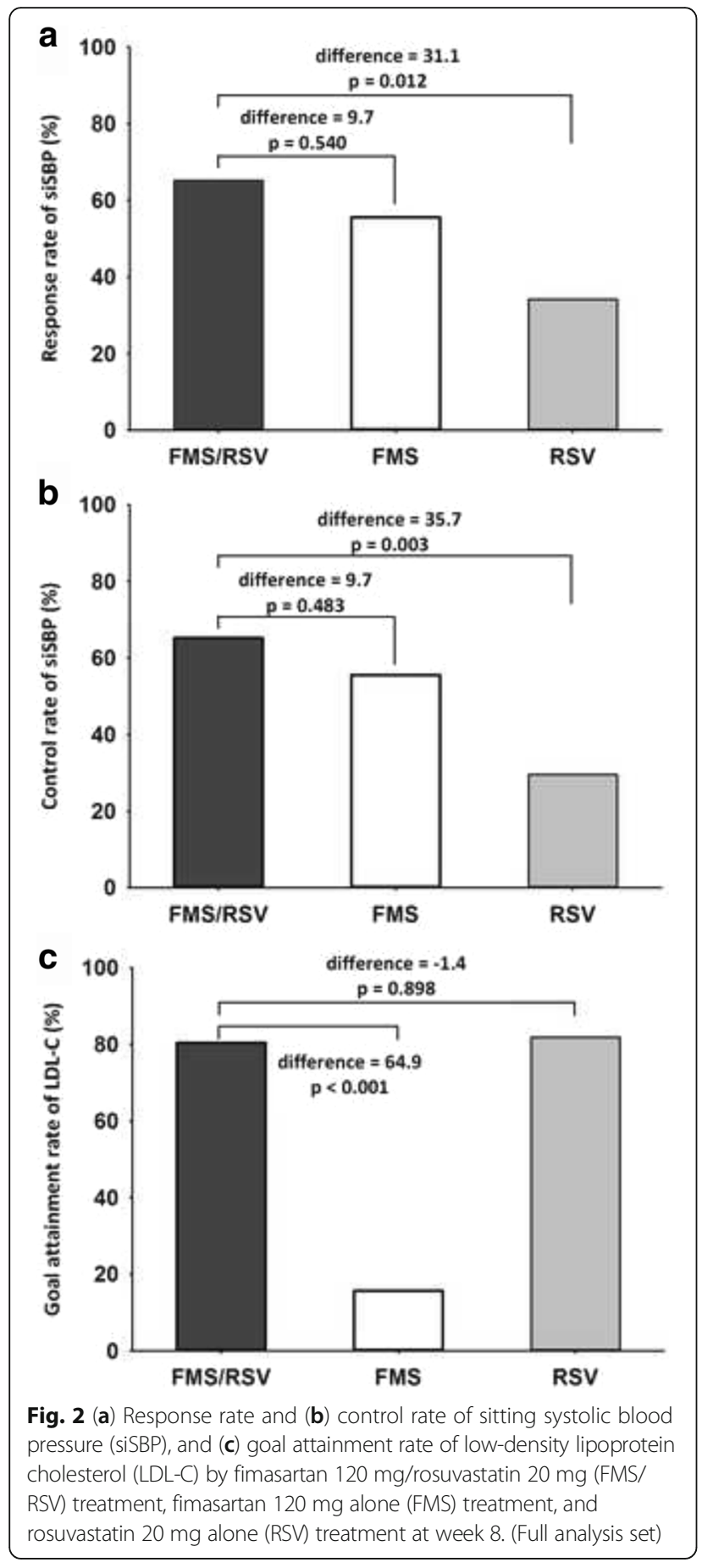

difference $=31.13 \%$, 95\% confidence interval 11.49 to $50.76, p=0.012)$. The control rate of siSBP in FMS/ RSV treatment, FMS alone treatment, and RSV alone treatment groups was $65.22,55.56$, and $29.55 \%$, respectively (FMS/RSV vs. RSV, difference $=35.67 \%$, 95\% confidence interval 16.41 to $54.94, p=0.003)$. The goal attainment rate of LDL-C in the FMS/RSV treatment, FMS alone treatment, and RSV alone treatment groups was $80.43,15.56$, and $81.82 \%$, respectively (FMS/RSV vs. FMS, difference $=64.88 \%$, 95\% confidence interval 49.27 to $80.49, p<0.001)$. In PPS analysis, the response and control rates of siSBP and siDBP, and the goal attainment rate of LDL-C were similar to those from FAS analysis (Table 4). The percentage of patients reaching their siSBP and LDL-C therapeutic goals after FMS/RSV treatment was $56.5 \%$, which was higher than with FMS alone treatment $(4.44 \%, p<0.001)$ or with RSV alone treatment (25.0\%, $p=0.003$ ) (Fig. 3).

Similar to changes in LDL-C, FMS/RSV treatment also showed a greater lowering effect on TC and triglyceride, as well as HDL-C elevation compared to that reported for FMS alone treatment (Table 5).

\section{Safety and tolerability}

In the safety set $(n=139)$ analysis, the incidence of adverse events considered to be related to the study drugs was $8.63 \%(n=12$, Table 6$)$. There was no significant difference in the incidence of study drug-related adverse events between treatment groups. Adverse events reported were dyspepsia $(n=1)$, nausea $(n=1)$, pyrexia $(n=1)$, and hepatitis $(n=1)$ in the FMS/RSV treatment group; upper abdominal pain $(n=1)$, elevation of hepatic enzyme $(n=1)$, and pollakiuria $(n=1)$ in the FMS alone treatment group; and headache $(n=2)$, hyperkalemia $(n=1)$, insomnia $(n=1)$, and pruritus $(n=1)$ in the RSV alone treatment group. There was no serious adverse event related to treatment with the study drugs.

\section{Discussion}

This study demonstrated that co-administration of FMS and RSV for 8 weeks to patients with hypertension and dyslipidemia was safe and effective in lowering blood pressure and LDL-C. The blood pressure-lowering effect of co-administration of FMS and RSV was not different from that of the FMS alone treatment, but significantly larger than that of the RSV alone treatment. The coadministration of FMS and RSV lowered LDL-C levels with similar effects as that of RSV alone treatment but significantly greater effects than that of FMS alone treatment. The response rates of siSBP and LDL-C upon coadministration of FMS and RSV were stronger than that after either RSV alone or FMS alone treatments.

Fimasartan is an antihypertensive drug that selectively blocks the angiotensin II type 1 receptor. It is used as a medication alone or in combination with other antihypertensive drugs [13, 14]. Its safety and efficacy have been proven $[15,17]$. Rosuvastatin, an inhibitor of HMG-CoA reductase, can reduce LDL-cholesterol more effectively than other statins [18] with proven evidence for CVD prevention [19]. The result of this study showed that there was no interference between fimasartan and rosuvastatin 
Table 4 Response rate and control rate of sitting systolic blood pressure, and goal attainment rate of low-density lipoprotein cholesterol by each treatment. (Analysis of per-protocol set)

\begin{tabular}{|c|c|c|c|c|c|c|c|}
\hline & \multicolumn{3}{|c|}{ Summary of each treatment } & \multicolumn{2}{|c|}{ FMS/RSV vs FMS } & \multicolumn{2}{|l|}{ FMS/RSV vs RSV } \\
\hline & $\overline{\text { FMS/RSV }}$ & FMS & RSV & Difference(SE) & $\overline{P \text {-value }}{ }^{\mathrm{d}}$ & Difference(SE) & $P$-value \\
\hline & $N=35$ & $N=36)$ & $N=37$ & (95\% C.I.) & & (95\% C.I.) & \\
\hline \multicolumn{8}{|l|}{ siSBP } \\
\hline Response rate ${ }^{\mathrm{a}}, \mathrm{n}(\%)$ & $26(74.29)$ & $23(63.89)$ & $15(40.54)$ & $10.40(10.89)$ & & $33.75(10.94)$ & \\
\hline $95 \% \mathrm{Cl}$ & $(59.81,88.77)$ & $(48.20,79.58)$ & $(24.72,56.36)$ & $(-10.95,31.75)$ & 0.390 & $(12.30,55.19)$ & 0.010 \\
\hline Control rate ${ }^{\mathrm{b}}, \mathrm{n}(\%)$ & $26(74.29)$ & $23(63.89)$ & $13(35.14)$ & $10.40(10.89)$ & & $39.15(10.78)$ & \\
\hline $95 \% \mathrm{Cl}$ & $(59.81,88.77)$ & $(48.20,79.58)$ & $(19.75,50.52)$ & $(-10.95,31.75)$ & 0.358 & $(18.03,60.28)$ & 0.003 \\
\hline \multicolumn{8}{|l|}{ LDL-C } \\
\hline Goal attainment rate ${ }^{c}, \mathrm{n}(\%)$ & $33(94.29)$ & $7(19.44)$ & $34(91.89)$ & $74.84(7.67)$ & & $2.39(5.96)$ & \\
\hline $95 \% \mathrm{Cl}$ & $(86.60,100.00)$ & $(6.52,32.37)$ & $(83.10,100.00)$ & $(59.80,89.88)$ & $<0.001$ & $(-21.13,25.12)$ & 0.082 \\
\hline
\end{tabular}

FMS/RSV fimasartan $120 \mathrm{mg} /$ rosuvastatin $20 \mathrm{mg}$ treatment, FMS fimasartan $120 \mathrm{mg}$ alone treatment, RSV rosuvastatin $20 \mathrm{mg}$ alone treatment, siSBP sitting systolic blood pressure, $L D L-C$ low-density lipoprotein cholesterol, $C I$ confidence interval, $S E$ standard error

${ }^{a}$ Response Rate of siSBP: proportion of the subjects whose siSBP $<140 \mathrm{mmHg}$ or Chang from Baseline of siSBP at Week $8 \geq 20 \mathrm{mmHg}$

${ }^{b}$ Control rate of siSBP: proportion of the subjects whose siSBP $<140 \mathrm{mmHg}$

'Goal attainment rate of LDL-C: proportion of the subjects whose LDL-C $<100 \mathrm{mg} / \mathrm{dL}$ (high risk), LDL-C $<130 \mathrm{mg} / \mathrm{dL}$ (moderate high or moderate risk),

LDL-C $<160 \mathrm{mg} / \mathrm{dL}$ (low risk)

${ }^{\mathrm{d} C o m p a r i s o n}$ between combination therapy and monotherapy was analyzed by Logistic regression model adjusted for baseline values, age, and gender, smoking status

on the efficacy and the safety of both drugs when they were simultaneously co-administered.

Although both antihypertensive drugs and statins have robust evidences of effect regarding the prevention of CVD, poor adherence to medications can reduce their effects in clinical practice $[8,20]$. Based on data analysis of enrollees in the Korean National Health Insurance system, poor adherence to treatment in patients with hypertension has been found to be associated with increased mortality and hospitalization [7]. Among the study population, the proportion of hypertensive patients with poor (cumulative medication adherence $50-80 \%$ ) and intermediate (cumulative medication adherence $<80 \%$ )

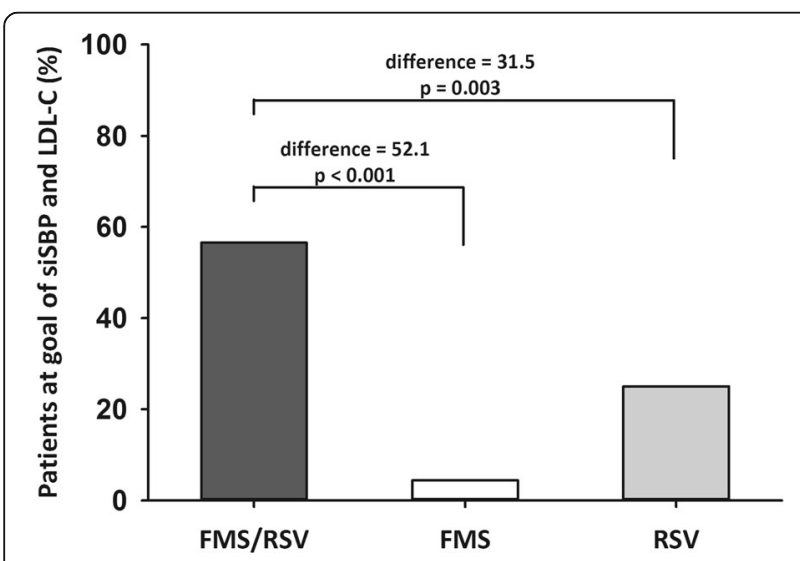

Fig. 3 Percentage of patients who reached sitting systolic blood pressure (siSBP) and low-density lipoprotein cholesterol (LDL-C) goals after fimasartan $120 \mathrm{mg} /$ rosuvastatin $20 \mathrm{mg}$ (FMS/RSV) treatment, fimasartan $120 \mathrm{mg}$ alone (FMS) treatment, or rosuvastatin $20 \mathrm{mg}$ alone (RSV) treatment at week 8 adherence to antihypertensive medications was more than $60 \%$. The association of long-term reduction of acute CVD events with high adherence to antihypertensive treatments was revealed based on the analysis of data obtained from 400 Italian primary care physicians [20], highlighting the importance of adherence to treatment in the prevention of CVD events.

Simplification of regimens by reducing the number of drugs prescribed and the frequency of dosing is an effective method to enhance patient's adherence to treatment [21, 22]. In a study evaluating patient adherence to hypertension medications, adherence to medication was inversely associated with the number of medications included in the regimen [23]. The levels of adherence to antihypertensive medications were $77.2 \%, 69.7 \%, 62.9 \%$, and $55.5 \%$ in patients receiving 1-, 2-, 3-, and 4-drug regimens respectively. Single pill combination can reduce the number of medications, and has been shown to improve patient adherence to treatment by reducing pill burden $[9,10]$. Single pill combination has improved compliance to medication by $21 \%$ and $26 \%$ compared to free-drug component regimen $[9,10]$.

A limitation of this study is that age and gender differences in blood pressure and LDL-C lowering due to different classes of antihypertensive drugs and rosuvastatin were not considered in the study design. Angiotensin converting enzyme inhibitors and beta blocker were more effective compared to calcium channel blockers and diuretics in lowering the blood pressure of young hypertensive patients (age 28-55 years) [24]. In older patients, calcium channel blockers and diuretics lowered blood pressure more than angiotensin converting enzyme 
Table 5 Least square mean changes in total cholesterol, high-density lipoprotein cholesterol and triglyceride from baseline after 8 weeks of treatment

\begin{tabular}{|c|c|c|c|c|c|c|c|}
\hline & \multicolumn{3}{|c|}{ Summary of each treatment } & \multicolumn{2}{|l|}{ FMS/RSV vs. FMS } & \multicolumn{2}{|l|}{ FMS/RSV vs. RSV } \\
\hline & $\begin{array}{l}\text { FMS/RSV } \\
(N=46)\end{array}$ & $\begin{array}{l}\text { FMS } \\
(N=45)\end{array}$ & $\begin{array}{l}\text { RSV } \\
(N=44)\end{array}$ & LSM difference (SE) & $P$-value ${ }^{a}$ & LSM difference (SE) & $P$-value ${ }^{a}$ \\
\hline Total cholesterol & $-36.13(1.77)$ & $-3.62(1.92)$ & $-35.99(1.95)$ & $-32.51(2.44)$ & & $-0.14(2.45)$ & \\
\hline $95 \% \mathrm{Cl}$ & $(-39.63,-32.64)$ & $(-7.41,0.17)$ & $(-39.86,-32.13)$ & $(-37.34,-27.69)$ & $<0.001$ & $(-4.99,4.71)$ & 0.954 \\
\hline $\mathrm{HDL}-\mathrm{C}$ & $10.24(2.63)$ & $-3.22(2.86)$ & $13.43(2.95)$ & $13.47(3.63)$ & & $-3.19(3.67)$ & \\
\hline $95 \% \mathrm{Cl}$ & $(5.03,15.45)$ & $(-8.88,2.43)$ & $(7.60,19.26)$ & $(6.28,20.65)$ & $<0.001$ & $(-10.46,4.08)$ & 0.387 \\
\hline Triglyceride & $-12.78(5.79)$ & $20.30(6.27)$ & $-13.65(6.41)$ & $-33.08(7.97)$ & & $0.88(8.06)$ & \\
\hline $95 \% \mathrm{Cl}$ & $(-24.24,-1.32)$ & $(7.89,32.71)$ & $(-26.34,-0.96)$ & $(-48.86,-17.30)$ & $<0.001$ & $(-15.08,16.83)$ & 0.914 \\
\hline
\end{tabular}

HDL-C high-density lipoprotein cholesterol, FMS/RSV fimasartan $120 \mathrm{mg} /$ rosuvastatin $20 \mathrm{mg}$ treatment, FMS fimasartan $120 \mathrm{mg}$ alone treatment, $R S V$ rosuvastatin $20 \mathrm{mg}$ alone treatment, $L S M$ least square mean, $S E$ standard error

${ }^{a}$ Comparison between the combination therapy and monotherapy was analyzed by ANCOVA model adjusted for baseline values, age, gender and smoking status

inhibitors [25]. A sex difference in blood pressure control has been suggested in animal studies, but not in human studies [26]. However, effect of age and gender differences of angiotensin receptor blockers including fimasartan have not been evaluated. The effect of age and gender differences of rosuvastatin in decreasing LDL-C is controversial. A pharmacokinetic study of rosuvastatin showed a small difference in plasma concentration between age and gender groups [27]. However, this difference was not considered clinically relevant because the difference was statistically insignificant [27]. On the other hand, rosuvastatin plasma levels were significantly higher in premenopausal compared with postmenopausal women [28]. The clinical significance of different plasma levels of rosuvastatin is unclear because there is no controlled study evaluating the effect of age and gender difference of rosuvastatin

Table 6 Incidence of drug related adverse events in safety analysis population

\begin{tabular}{lllll}
\hline Drug related adverse & \multicolumn{3}{l}{ Number (\%) of subjects with ADRs } & \\
\cline { 2 - 4 } & FMS/RSV & MFS & RSV & p-value \\
\hline Total number (\%) & $4(8.33)$ & $3(6.52)$ & $5(11.11)$ & 0.755 \\
Abdominal pain upper & - & $1(2.17)$ & - & \\
Dyspepsia & $1(2.08)$ & - & - & \\
Nausea & $1(2.08)$ & - & - & \\
Headache & - & - & $2(4.44)$ & \\
Pyrexia & $1(2.08)$ & - & - & \\
Hepatitis & $1(2.08)$ & - & - & \\
Hepatic enzyme increased & - & $1(2.17)$ & - & \\
Hyperkalaemia & - & - & $1(2.22)$ & \\
Insomnia & - & - & $1(2.22)$ & \\
Pollakiuria & - & $1(2.17)$ & - & \\
Pruritus & - & - & $1(2.22)$ & \\
\hline
\end{tabular}

ADRs adverse drug reactions, FMS/RSV fimasartan $120 \mathrm{mg} /$ rosuvastatin $20 \mathrm{mg}$ treatment, FMS fimasartan $120 \mathrm{mg}$ alone treatment, RSV rosuvastatin $20 \mathrm{mg}$ alone treatment treatment in lowering LDL-C. Although this study was designed without considering age and gender difference, ANCOVA model showed the age and gender independent efficacy of co-administered fimasartan and rosuvastatin.

\section{Conclusion}

The results of this study demonstrated that co-administration of fimasartan and rosuvastatin to patients with hypertension and hypercholesterolemia was efficacious and safe. Therefore, a single pill combination of both drugs is expected to be a suitable strategy for prevention of CVD events.

\section{Additional file}

Additional file 1: Table S1. List of Institutional Review Boards. (DOCX $18 \mathrm{~kb}$ )

\section{Abbreviations}

ANCOVA: Analysis of covariance; CVD: Cardiovascular disease; FAS: Full analysis set; FMS: Fimasartan 120 mg; FMS/RSV: Fimasartan 120 mg/ rosuvastatin $20 \mathrm{mg}$; HDL-C: High density lipoprotein cholesterol; HMGCoA: 3-hydroxy-3-methylglutaryl coenzyme A; LDL-C: Low density lipoprotein cholesterol; NCEP-ATP III: National Cholesterol Education Program Adult Panel III; PPS: Per-protocol set; RSV: Rosuvastatin 20 mg; SBP: Systolic blood pressure; siDBP: Sitting diastolic blood pressure; siSBP: Sitting systolic blood pressure; TC: Total cholesterol; TLC: Therapeutic life changes

\section{Acknowledgments}

We are thankful to Boryung Pharmaceutical Co. Ltd financial support and data analysis. We also thank MS. Seung Hee Jeong of Boryung Pharmaceutical Co. Ltd for data analysis.

\section{Funding}

This study was initiated and financially supported by Boryung Pharmaceutical Co. Ltd., Seoul, Republic of Korea (BR-FRC-CT-301). The sponsor supported the supply of study drug, laboratory test, data collection, and data analysis. The funding body had no role in data interpretation and the writing of the manuscript based on the data.

Availability of data and materials

Raw data of this study are available from the Boryung Pharmaceutical Co. Ltd. 


\section{Authors' contributions}

All authors acted as principal investigators at study sites, recruited patients, and collected data. Moo-Yong Rhee wrote the full manuscript. All authors reviewed and approved the final manuscript.

\section{Competing interests}

Dr. Rhee has received lecture honoraria from Pfizer Inc., LG Life Sciences Ltd, Bayer Korea Ltd., Hanmi Pharm. Co. Ltd., Yuhan Co. Ltd., Boryung Pharmaceutical Co. Ltd., and research grant from Boryung Pharmaceutical Co. Ltd. and Dong-A Pharmaceutical Co., Ltd.

Dr. Oh has received research grant from Boryung Pharmaceutical Co. Ltd. The authors have indicated that they have no other conflicts of interest with regard to the content of this article.

The study drugs were supplied by Boryung Pharmaceutical Co. Ltd. ClinicalTrials.gov Identifier: NCT02166814.

\section{Consent for publication}

Not applicable.

\section{Ethics approval and consent to participate}

The Ministry of Food and Drug Safety and Institutional Review Board of participating institution approved the study design.

\section{Author details}

${ }^{1}$ Cardiovascular Center, Dongguk University Ilsan Hospital, Goyang, Republic of Korea. ${ }^{2}$ Division of Cardiology, Gachon University Gil Hospital, Incheon, Republic of Korea. ${ }^{3}$ Division of Cardiology, Seoul St. Mary's Hospital, Seoul, Republic of Korea. ${ }^{4}$ Department of Internal Medicine, Kyungpook National University School of Medicine, Daegu, Republic of Korea. ${ }^{5}$ Division of Cardiology, Inje University Busan Paik Hospital, Busan, Republic of Korea. ${ }^{6}$ Division of Cardiology, Korea University Anam Hospital, Seoul, Republic of Korea. ${ }^{7}$ Department of Cardiology, Dankook University Hospital, Cheonan, Republic of Korea. ${ }^{8}$ Department of Internal Medicine, Daegu Catholic University Hospital, Daegu, Republic of Korea. ${ }^{9}$ Division of Cardiology, Dongguk University Gyeongju Hospital, Gyeongju, Republic of Korea. ${ }^{10}$ Department of Internal Medicine, Dong-A University College of Medicine, Busan, Republic of Korea. "1Department of Internal Medicine, Seoul National University College of Medicine, Seoul, South Korea. ${ }^{12}$ Division of Cardiology, Samsung Medical Center, Sungkyunkwan University School of Medicine, Seoul, Republic of Korea. ${ }^{13}$ Division of Cardiology, Seoul National University Hospital, Seoul, Republic of Korea. ${ }^{14}$ Department of Cardiology, Ajou University Hospital, Suwon, Republic of Korea. ${ }^{15}$ Division of Cardiology, Gangnam Severance Hospital, Seoul, Republic of Korea. ${ }^{16}$ Division of Cardiology, Severance Cardiovascular Hospital, Seoul, Republic of Korea. ${ }^{17}$ Department of Cardiology, Yeungnam University Hospital, Daegu, Republic of Korea. ${ }^{18}$ Division of Cardiology, Korea University Guro Hospital, 148 , Gurodong-ro, Guro-gu, Seoul 08308, Republic of Korea. ${ }^{19} \mathrm{Cardiac}$ and Vascular Center, Inje University Ilsan Paik Hospital, Goyang, Republic of Korea. ${ }^{20}$ Department of Cardiology, Inje University Haeundae Paik Hospital, Busan, Republic of Korea. ${ }^{21}$ Department of Cardiology, Inha University Hospital, Incheon, Republic of Korea. ${ }^{22}$ Division of Cardiology, Jeju National University Hospital, Jeju, Republic of Korea. ${ }^{23}$ Department of Cardiovascular, Chonnam National University Hospital, Gwangju, Republic of Korea. ${ }^{24}$ Division of Cardiology, Chungnam National University Hospital, Daejeon, Republic of Korea. ${ }^{25}$ Division of Cardiology, Department of Medicine, Kangbuk Samsung Hospital, Sungkyunkwan University School of Medicine, Seoul, Republic of Korea. ${ }^{26}$ Department of Cardiology, Seoul Medical Center, Seoul, Republic of Korea. ${ }^{27}$ Division of Cardiology, Seoul Metropolitan Government Seoul National University Hospital Boramae Medical Center, Seoul, Republic of Korea. ${ }^{28}$ Cardiovascular Center, Pusan National University Yangsan Hospital, Yangsan, Republic of Korea.

\section{Received: 11 August 2016 Accepted: 13 December 2016} Published online: 05 January 2017

\section{References}

1. Johnson ML, Pietz K, Battleman DS, Beyth RJ. Prevalence of comorbid hypertension and dyslipidemia and associated cardiovascular disease. Am J Manag Care. 2004;10:926-32.

2. Neaton JD, Wentworth D. Serum cholesterol, blood pressure, cigarette smoking, and death from coronary heart disease. Overall findings and differences by age for 316,099 white men. Multiple Risk Factor Intervention Trial Research Group. Arch Intern Med. 1992;152:56-64.

3. Kannel WB. Fifty years of Framingham Study contributions to understanding hypertension. J Hum Hypertens. 2000;14:83-90.

4. Mancia G, Fagard R, Narkiewicz K, Redon J, Zanchetti A, Bohm M, et al. 2013 ESH/ESC Guidelines for the management of arterial hypertension: the Task Force for the management of arterial hypertension of the European Society of Hypertension (ESH) and of the European Society of Cardiology (ESC). J Hypertens. 2013;31:1281-357.

5. Expert Panel on Detection E, Treatment of High Blood Cholesterol in A. Executive Summary of The Third Report of The National Cholesterol Education Program (NCEP) Expert Panel on Detection, Evaluation, And Treatment of High Blood Cholesterol In Adults (Adult Treatment Panel III). JAMA. 2001;285:2486-97.

6. Emberson J, Whincup P, Morris R, Walker M, Ebrahim S. Evaluating the impact of population and high-risk strategies for the primary prevention of cardiovascular disease. Eur Heart J. 2004;25:484-91.

7. Kim S, Shin DW, Yun JM, Hwang Y, Park SK, Ko YJ, et al. Medication Adherence and the Risk of Cardiovascular Mortality and Hospitalization Among Patients With Newly Prescribed Antihypertensive Medications. Hypertension. 2016;67:506-12.

8. Simpson SH, Eurich DT, Majumdar SR, Padwal RS, Tsuyuki RT, Varney J, et al. A meta-analysis of the association between adherence to drug therapy and mortality. BMJ. 2006:333:15.

9. Gupta AK, Arshad S, Poulter NR. Compliance, safety, and effectiveness of fixed-dose combinations of antihypertensive agents: a meta-analysis. Hypertension. 2010;55:399-407.

10. Bangalore S, Kamalakkannan G, Parkar S, Messerli FH. Fixed-dose combinations improve medication compliance: a meta-analysis. Am J Med. 2007;120:713-9.

11. National Cholesterol Education Program Expert Panel on Detection E, Treatment of High Blood Cholesterol in A. Third Report of the National Cholesterol Education Program (NCEP) Expert Panel on Detection, Evaluation, and Treatment of High Blood Cholesterol in Adults (Adult Treatment Panel III) final report. Circulation. 2002;106:3143-421.

12. Coleman A, Freeman P, Steel S, Shennan A. Validation of the Omron 705IT (HEM-759-E) oscillometric blood pressure monitoring device according to the British Hypertension Society protocol. Blood Press Monit. 2006;11:27-32.

13. Rhee MY, Baek SH, Kim W, Park CG, Park SW, Oh BH, et al. Efficacy of fimasartan/hydrochlorothiazide combination in hypertensive patients inadequately controlled by fimasartan monotherapy. Drug Des Devel Ther. 2015:9:2847-54

14. Lee HY, Kim YJ, Ahn T, Youn HJ, Chull Chae S, Seog Seo H, et al. A Randomized, Multicenter, Double-blind, Placebo-controlled, 3 × 3 Factorial Design, Phase II Study to Evaluate the Efficacy and Safety of the Combination of Fimasartan/Amlodipine in Patients With Essential Hypertension. Clin Ther. 2015;37:2581-96. e3.

15. Lee H, Yang HM, Lee HY, Kim JJ, Choi DJ, Seung KB, et al. Efficacy and tolerability of once-daily oral fimasartan 20 to $240 \mathrm{mg} / \mathrm{d}$ in Korean Patients with hypertension: findings from Two Phase II, randomized, double-blind, placebo-controlled studies. Clin Ther. 2012;34:1273-89.

16. Olsson AG, Pears J, McKellar J, Mizan J, Raza A. Effect of rosuvastatin on low-density lipoprotein cholesterol in patients with hypercholesterolemia. Am J Cardiol. 2001;88:504-8.

17. Lee SE, Kim YJ, Lee HY, Yang HM, Park CG, Kim JJ, et al. Efficacy and tolerability of fimasartan, a new angiotensin receptor blocker, compared with losartan (50/100 mg): a 12-week, phase III, multicenter, prospective, randomized, double-blind, parallel-group, dose escalation clinical trial with an optional 12-week extension phase in adult Korean patients with mild-to-moderate hypertension. Clin Ther. 2012;34:552-68. 68 e1-9.

18. Jones PH, Davidson MH, Stein EA, Bays HE, McKenney JM, Miller E, et al. Comparison of the efficacy and safety of rosuvastatin versus atorvastatin, simvastatin, and pravastatin across doses (STELLAR* Trial). Am J Cardiol. 2003;92:152-60.

19. Ridker PM, Danielson E, Fonseca FA, Genest J, Gotto Jr AM, Kastelein JJ, et al. Rosuvastatin to prevent vascular events in men and women with elevated C-reactive protein. N Engl J Med. 2008;359:2195-207.

20. Mazzaglia G, Ambrosioni E, Alacqua M, Filippi A, Sessa E, Immordino V, et al. Adherence to antihypertensive medications and cardiovascular morbidity among newly diagnosed hypertensive patients. Circulation. 2009;120:1598-605. 
21. Claxton AJ, Cramer J, Pierce C. A systematic review of the associations between dose regimens and medication compliance. Clin Ther. 2001;23:1296-310.

22. Mateo JF, Gil-Guillen VF, Mateo E, Orozco D, Carbayo JA, Merino J. Multifactorial approach and adherence to prescribed oral medications in patients with type 2 diabetes. Int I Clin Pract. 2006;60:422-8.

23. Fung V, Huang J, Brand R, Newhouse JP, Hsu J. Hypertension treatment in a medicare population: adherence and systolic blood pressure control. Clin Ther. 2007;29:972-84.

24. Deary AJ, Schumann AL, Murfet H, Haydock SF, Foo RS, Brown MJ. Double-blind, placebo-controlled crossover comparison of five classes of antihypertensive drugs. J Hypertens. 2002;20:771-7.

25. ALLHAT Officers and Coordinators for the ALLHAT Collaborative Research Group. The Antihypertensive and Lipid-Lowering Treatment to Prevent Heart Attack Trial. Major outcomes in high-risk hypertensive patients randomized to angiotensin-converting enzyme inhibitor or calcium channel blocker vs diuretic: The Antihypertensive and Lipid-Lowering Treatment to Prevent Heart Attack Trial (ALLHAT). JAMA. 2002;288:2981-97.

26. Maranon R, Reckelhoff JF. Sex and gender differences in control of blood pressure. Clin Sci (Lond). 2013;125:311-8.

27. Martin PD, Dane AL, Nwose OM, Schneck DW, Warwick MJ. No effect of age or gender on the pharmacokinetics of rosuvastatin: a new HMG-CoA reductase inhibitor. J Clin Pharmacol. 2002;42:1116-21.

28. Nazir S, labal Z, Nasir F. Impact of menopause on pharmacokinetics of rosuvastatin compared with premenopausal women. Eur J Drug Metab Pharmacokinet. 2016;41:505-9.

\section{Submit your next manuscript to BioMed Central} and we will help you at every step:

- We accept pre-submission inquiries

- Our selector tool helps you to find the most relevant journal

- We provide round the clock customer support

- Convenient online submission

- Thorough peer review

- Inclusion in PubMed and all major indexing services

- Maximum visibility for your research

Submit your manuscript at www.biomedcentral.com/submit 